\title{
Adaptive Tolerant State Estimation under Model Uncertainty in Power Systems
}

\author{
Ruizi Ma
}

check for

updates

Citation: Ma, R. Adaptive Tolerant State Estimation under Model Uncertainty in Power Systems. Energies 2021, 14, 2111. https:// doi.org/10.3390/en14082111

Academic Editors: Victor Becerra and Ahmed Abu-Siada

Received: 21 February 2021

Accepted: 6 April 2021

Published: 9 April 2021

Publisher's Note: MDPI stays neutral with regard to jurisdictional claims in published maps and institutional affiliations.

Copyright: (C) 2021 by the author. Licensee MDPI, Basel, Switzerland. This article is an open access article distributed under the terms and conditions of the Creative Commons Attribution (CC BY) license (https:// creativecommons.org/licenses/by/ $4.0 /)$.
College of Mechanical and Electrical Engineering, China Jiliang University, Hangzhou 310018, China; ruizi.ma@cjlu.edu.cn

\begin{abstract}
In this paper an adaptive tolerant estimator using singular value decomposition is proposed for a distribution network under model uncertainty in power systems. The adaptive tolerant estimator was designed with adjusted parameters and adjusted weights to overcome the limitations of model uncertainty. The estimator that reduces the measurement errors is adaptive to fast parameter changes in complicated environments. The singular value decomposition method was combined into the state estimator, which extended the over-determined cases to under-determined cases under model uncertainty. The performance of the tolerant estimator was compared with the conventional adaptive estimator, and the tolerant estimator showed accurate estimations against model uncertainty in complicated measurement environments.
\end{abstract}

Keywords: singular value decomposition; power systems; state estimator; adaptive; under-determined

\section{Introduction}

State estimation (SE) is important for energy management to ensure the stability and quality of electrical power networks. In state estimation, redundant measurements are captured by the traditional supervisory control and data acquisition (SCADA) systems with advanced synchronized phasor measurement units (PMUs). These methods are development for the estimator to measure the state vectors, which include voltage magnitudes and angles at all buses in the power system [1,2]. The states in the power system are estimated by a state estimator. State estimators provide reliable real-time data for the power system, including that of the existing state. The estimation data uses security assessment functions to analyze contingencies and reliably determine any required corrective actions for the power systems in distribution networks [3].

Different estimation methods have been used to estimate state vectors in electric power grids, such as robust estimation and optimization algorithms. The algebraic technique to transform the power flows into rank-one measurements has been proposed for robust state estimation [4]. The iterated extended Kalman filter was designed for robust dynamic state estimation [5]. A fully distributed robust bilinear state-estimation method is applicable to multi-area power systems with nonlinear measurements [6]. Particle swarm optimization [7] and graph theory [8,9] have been employed to optimize the parameters for state estimation.

Compared to other conventional estimation algorithms, the weighted least square (WLS) method with fast convergence properties is widely applied to estimate the state vectors in the distribution network. WLS is a static state estimator that obtains results from the current measurement data without recent historical information, with the exception of some pseudo-measures [10]. The estimator based on the static measurement model and collected data is considered to be time-invariant. The analysis of conventional SE using the WLS approach in the power system is illustrated in detail in Schweppe et al. [11]. Static SE based on WLS algorithms has been applied in wide areas of the power system, such as integrated power systems [12], HVDC (High Voltage Direct Current Transmission) systems [13], and power electronic devices [14]. 
In static state estimation problems, the measurement parameters are generally considered to be unadjusted without considering model uncertainties, even in complicated measurement environments. However, the parameters can change quickly within a large range due to various factors $[15,16]$ and sudden power failures $[17,18]$ in complicated environments. For example, a harmonic current is produced from power electronic devices in power systems. Harmonic frequencies have impacts on the line transposition, as illustrated in Arrillaga et al. [19]. Temperature variations cause changes in conductor resistance; in particular, the series resistance depending on temperature results in line sag on hot days [20,21]. Furthermore, complicated measurement environments lead to significant time delay, which can result in the failure of measurement data to correct for fast parameter changes. This reduces the accuracy of the state estimator, which aggravates measurement errors. As a result, the parameter data acquisition in the control center deviates from the real data, and fast parameter changes are not able to be corrected for in the estimation systems under complicated transmission conditions.

Furthermore, conventional state estimation depends on the inverse of the Jacobian matrix, which is not obtained in over-determined cases with rectangular singular matrices. The solutions involve the calculation of Moore-Penrose inverse, but this is constrained such that the column rank of the matrices is not deficient. When the estimation states are not fully observed, the singular value decomposition (SVD) approach [22] is employed to generate the Moore-Penrose inverse, although the matrices are column deficient. The SVD technique, which inverts the measurement model with non-fully observable states [23], was introduced to estimate states in the under-determined cases.

To ensure the robustness of state estimation, robust estimators have been designed; for example, the maximum correntropy criterion with Laplace kernel function can be employed as the objective function of state estimation to design a robust estimator on the basis of a lower order correction equation [24]. A systematic approach computes the optimal rotation angles and scaling factors of the WLAV-T, which ensures that SE is not sensitive to bad measurements by mitigating the effect of leverage points [25].

In our paper observability analysis is used to demonstrated that the adaptive state estimator is insensitive to major deviations in the limit numbers of redundant measurements. Furthermore, we strengthened the robustness against poor data in the measurements by combining a tolerant measurement model into the WLS algorithms, thus improving the robustness via the processing of adjustable parameters and adjustable weights. Furthermore, the WLS algorithms were developed to be adaptive, rather than to formulate the mean square errors of the estimation in minimization. The iterations ensure state convergence by minimizing the residual mean square errors, which strengthens the robustness of WLS.

An adaptive tolerant estimator with model uncertainty is proposed to overcome the fast parameter changes in the power systems under complicated measurement conditions. We designed an adaptive tolerant measurement model, which comprises adjustable parameters and weights to reduce the state estimation errors caused by the fast parameter changes in complicated measurement environments. The adaptive tolerant estimator overcomes the limitations of fast temperature variations and serious transmission delay by the design of adaptive tolerant measurement models. The adjusted weights are compensated for the deviations caused by the measurement devices error that are not included in the parameter changes under the complicated measurement conditions. The introduction of the singular value decomposition method extends the estimation from over-determined cases to under-determined cases that do not rely on fully observable states. Although measurement data are not obtained from unobservable states, the mapping and transformation property of SVD realizes full state estimations with model uncertainty in the complicated measurement environments.

This paper is organized as follows. Section 2 illustrates the conventional WLS state estimation. The adaptive tolerant estimator using SVD is demonstrated in Section 3. The measurement residuals and observability analysis are demonstrated in Section 4. Section 5 shows the simulation results. Finally, the conclusions are given in Section 6. 


\section{WLS State Estimation}

The aim of conventional state estimation is to optimize estimations of the voltage phasors at all buses with a set of measurement data using the current errors. A mathematical model $h(x)$ that describes the functional relations between measurement vector $z$, state variable vector $x$, and measurement error $e$ is given by Equation (1) [2].

$$
z=h(x)+e
$$

where $x \in R^{n}$ is the state vector, $n$ represents the number of state variables; $z \in R^{m}(m>n)$ is the measurement vector; $h(x) \in R^{m}$ relates the state variables to measurement data; $e \in R^{m}$ is the measurement error vector, assuming a Gaussian distribution error with distribution parameters of mean 0 and standard deviation of $\sigma_{i} i=1,2, \ldots \mathrm{m}$.

The weighted least squares (WLS) state estimation problem is solved by minimizing the objective function described in Equation (2).

$$
J(x)=\sum_{i=1}^{m} \frac{\left(z_{i}-h_{i}(x)\right)^{2}}{R_{i i}}=[z-h(x)]^{T} R^{-1}[z-h(x)]
$$

where $R=\operatorname{diag}\left\{\sigma_{1}^{2}, \sigma_{2}^{2}, \ldots, \sigma_{m}^{2}\right\}$ is the measurement covariance matrix and the standard deviation $\sigma_{i}$ reflects the desired accuracy. To obtain the solution of Equation (2) at the minimum, the first-order optimality conditions for $J(x)$ will be satisfied in the form of Equation (3).

$$
g(x)=\frac{\partial J(x)}{\partial x}=-H^{T}(x) R^{-1}[z-h(x)]=0
$$

where $H(x)=\left[\frac{\partial h(x)}{\partial x}\right]$, which is the Jacobian matrix, is a sensitive matrix containing the state variables. The result of the Jacobian for an observable power system has rank $n$, which is equal to the number of state variables. Equation (3) is transformed into Equation (4) by the Taylor series expansion around the state $x^{k}$, as follows:

$$
\left[G\left(x^{k}\right)\right] \Delta x^{k+1}=H^{T}\left(x^{k}\right) R^{-1}\left[z-h\left(x^{k}\right)\right]
$$

where $G(x)=\frac{\partial g(x)}{\partial x}=H^{T}(x) R^{-1} H(x)$ is the gain matrix, $\Delta x^{k+1}=x^{k+1}-x^{k}$.

The iterative solution of Equation (4) for WLS state estimation is obtained using the Gauss-Newton method. The Gauss-Newton iteration step is given as:

$$
x^{k+1}=x^{k}+G\left(x^{k}\right)^{-1} H^{T}\left(x^{k}\right) R^{-1}\left[z-h\left(x^{k}\right)\right]
$$

The iterative algorithm repeats until the predefined value of $\Delta x$ is achieved. $h(x)$ is a static measurement model, and obtains network information from the historic data in a steady measurement environment. Traditional state estimation using the static model is constrained when state variables are not fully determined and parameters are subject to rapid changes. To overcome these limitations, an adaptive tolerant WLS state estimation method under uncertain measurement conditions in a complicated environment is introduced in the next section.

\section{Adaptive Tolerant Estimator Using SVD}

\subsection{Adaptive Tolerant Measurement Model with Weight Adjustment}

To solve the problem of model uncertainty arising from fast parameter changes, an adaptive tolerant measurement model for state estimation is designed in this section. The tolerant estimator overcomes the constraints of fast temperature variations and significant transmission delay in complicated measurement environments. The adaptive tolerant model containing adjustable parameters and adjustable weights reduces measurement 
errors when the measurement data are not able to be updated quickly enough to capture the fast changes of measurement conditions.

Due to the unpredictable factors of large temperature variations and significant transmission delay in complicated environments, line parameters change within a range and cannot be corrected by supervisory control and data acquisition systems. To solve this problem, we assumed the adjusted safety allowance admittance shunted in the base admittance. The equivalent circuit transformed from the real network is shown in Figure 1.

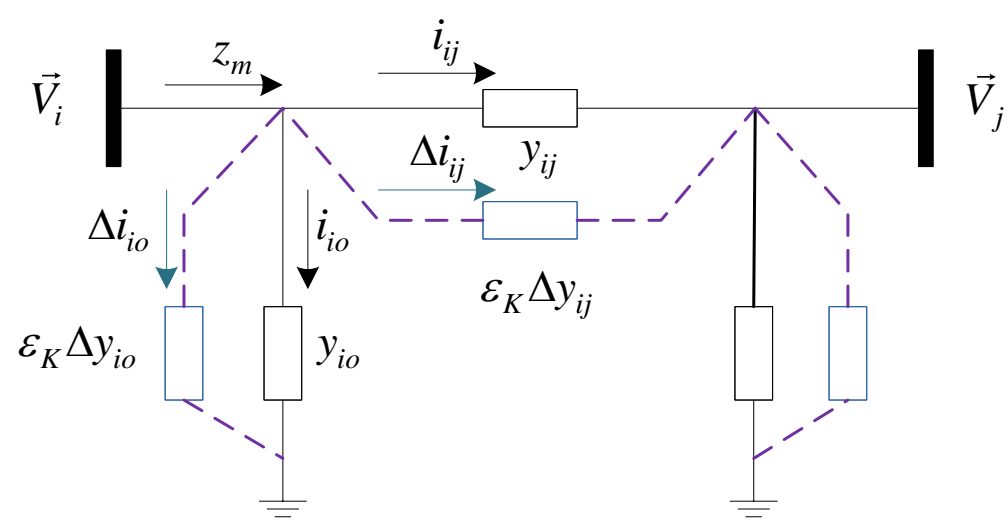

Figure 1. Equivalent circuit with adjustable admittances.

In Figure $1, y_{i j}$ and $y_{i o}$ are constant admittances representing the nominal value, $i_{i j}$ and $i_{i o}$ represent the currents following through $y_{i j}$ and $y_{i o}$, and $\varepsilon_{K} \in[-1,1]$ refers to the adaptive parameters. According to the temperature variations, $\Delta y_{i j}$ and $\Delta y_{i o}$ are safety allowance admittances approximated by the previous static data; $\varepsilon_{K} \Delta y_{i j}$ and $\varepsilon_{K} \Delta y_{i o}$ are the adjusted terms; and $\Delta i_{i j}$ and $\Delta i_{i o}$ represent the currents following through $\varepsilon_{K} \Delta y_{i j}$ and $\varepsilon_{K} \Delta y_{i o}$. Due to the transmission delay, $\varepsilon_{K}$ will be adaptive to be values within the range. $\varepsilon_{K} \Delta y_{i j}$ and $\varepsilon_{K} \Delta y_{i o}$ will compensate for the small changes of line parameters with model uncertainty caused by large temperature variations and significant transmission delay. Accordingly, Equation (6) is obtained from Figure 1.

$$
z_{m}=i_{i j}+\Delta i_{i j}+i_{i o}+\Delta i_{i o}
$$

Correspondingly, the tolerant measurement equation is given as:

$$
\begin{aligned}
z & =h_{\Sigma, \Delta}(x)+e_{\Sigma, \Delta} \\
& =h_{\Sigma, \Delta}(x)+e+\Delta d
\end{aligned}
$$

where $h_{\Sigma, \Delta}(x)$ represents the relationship between state variables and measurement values and comprises adjusted admittances. The measurement model is constructed with adjustable network parameters, so $h_{\Sigma, \Delta}(x)$ includes an adjustable section to amend the main measurement model $h(x)$. $e$ denotes the basis errors of the measurement devices; $\Delta d$ denotes to the deviations of the adjusted term caused by the model uncertainties; $e_{\Sigma, \Delta}=e+\Delta d$ represents the equivalent measurement errors in the data-tolerant measurement model. According to the weighted algorithm [26], we assume that $e$ and $\Delta d$ are independent. To demonstrate the levels of the individual measurements, the equivalent standard deviation matrix can be approximated as:

$$
R_{z, e q}=R_{z}+R_{d}
$$

where $R_{z, e q}$ consists of two independent components, $R_{z}$ and $R_{d} . R_{z}=\operatorname{diag}\left\{\sigma_{1}, \sigma_{2}, \ldots, \sigma_{m}\right\}$ represents the respective standard deviation matrix of $e$ and $R_{d}$ denotes the respective 
standard deviation matrix of $\Delta d$, assuming the variation of $\Delta d$ is uniformly distributed and its standard deviation matrix is given by:

$$
R_{d}=\operatorname{Diag}\left\{\left(\frac{1}{12}\left(\Delta \bar{d}_{1}-\Delta \underline{d}_{1}\right)^{2}\right)^{\frac{1}{2}} \ldots\left(\frac{1}{12}\left(\Delta \bar{d}_{m}-\Delta \underline{d}_{m}\right)^{2}\right)^{\frac{1}{2}}\right\}
$$

where $\Delta \underline{d}_{i}$ and $\Delta \bar{d}_{i} i=1,2, \ldots m$ denote the lower and upper bounds of $\Delta d_{i}$. The individual errors are weighted with the adjusted equivalent standard deviation $R_{z, e q}$. The adaptive tolerant state estimation is demonstrated in the next section. Equation (2) can be minimized for over-determined cases, and thus converges to the same solution as the traditional state estimation. Compared to the traditional state estimation algorithms, both the partially and fully under-determined cases can be solved using the adaptive WLS state estimation with the data-tolerant measurement model, as shown in following sections.

\subsection{Distribution State Estimation Based on Adaptive Tolerant Measurement Model Using SVD}

The traditional state estimator is only applied to over-determined cases, thus, the measurement numbers must be greater than the state numbers. The SVD algorithms extend the over-determined case to the under-determined case [27,28]. Due to the constraints, an adaptive tolerant estimator using SVD is proposed in this section to extend the traditional WLS to the under-determined cases under the condition of fast parameter changes in a complicated measurement environment. The adaptive state estimation technique does not rely on the observability of all state variables in the distribution network, and replaces this with a direct mapping of the available input data to the determined output data.

The sensitivity matrix $H_{\Sigma, \Delta} x$ of the dynamic measurement model $h_{\Sigma, \Delta}(x)$ with an auxiliary adjusted part is defined as:

$$
H_{\Sigma, \Delta}(x)=\frac{\partial h_{\Sigma, \Delta}(x)}{\partial x}
$$

The state error $\Delta x^{k+1}$ with the sensitivity matrix $H_{\Sigma, \Delta} x$ in the adaptive tolerant measurement model is used as the residual estimation error in the $k t h$ iteration, defined as Equation (11).

$$
H_{\Sigma, \Delta}(x) \Delta x^{k+1}=\left[z-h_{\Sigma, \Delta}(x)\right]
$$

The standard deviation indicates the quality of individual measurement. Weighting the standard deviation in the adaptive tolerant measurement model under the condition of complicated environment, we obtain Equation (12).

$$
\left(R_{z}+R_{d}\right)^{-1} H_{\Sigma, \Delta}(x) \Delta x^{k+1}=\left(R_{z}+R_{d}\right)^{-1}\left[z-h_{\Sigma, \Delta}(x)\right]
$$

To replace the inverse for the term of $\left(R_{z}+R_{d}\right)^{-1} H_{\Sigma, \Delta}(x)$, the SVD technique is employed to obtain the Moore-Penrose inverse, as Equation (13).

$$
\Upsilon^{\dagger}(x)=\left[\left(R_{z}+R_{d}\right)^{-1} H_{\Sigma, \Delta}(x)\right]^{\dagger}
$$

The iterative step based on the Newton iteration method using the adaptive tolerant measurement model is correspondingly adjusted as:

$$
\Delta x^{k+1}=\left[\left(R_{z}+R_{d}\right)^{-1} H_{\Sigma, \Delta}(x)\right]^{\dagger}\left(R_{z}+R_{d}\right)^{-1}\left[z-h_{\Sigma, \Delta}(x)^{k}\right]
$$

The SVD method provides the Moore-Penrose inverse to overcome the limitation of the existence of $G\left(x^{k}\right)^{-1}$, because without an existing constraint condition, the MoorePenrose inverse ensures that the inverse always exists. Therefore, the iterative step is 
able to operate smoothly. Then, the iteration cycle equation using the adaptive tolerant measurement model is given as:

$$
x^{k+1}=x^{k}+\left[\left(R_{z}+R_{d}\right)^{-1} H_{\Sigma, \Delta}(x)\right]^{\dagger}\left(R_{z}+R_{d}\right)^{-1}\left[z-h_{\Sigma, \Delta}(x)^{k}\right]
$$

The advantage function of SVD can transform the matrix $\Upsilon(x)$ to the factorization form, which comprises a left singular matrix $V_{\curlyvee}(x)$, singular value matrix $\Sigma_{\Upsilon}(x)$, and right singular matrix $U_{\curlyvee}(x)$. Then, the SVD of $\Upsilon(x)$ is given as Equation (16).

$$
\Upsilon(x)=V_{\curlyvee}(x) \Sigma_{\curlyvee}(x) U_{\curlyvee}^{T}(x)
$$

where $V_{\curlyvee}(x) \in R^{m \times m}$ is a complex unitary matrix, which comprises a set of left singular vectors $V_{\curlyvee, i}(x) i=1,2, \ldots, m$. spanning the entire measurement space. $U_{\curlyvee}(x) \in R^{n \times n}$ is a complex unitary matrix, which comprises a set of right singular vectors $U_{\curlyvee, i}(x) i=1,2, \ldots, n$. spanning the entire state space.

The singular matrix $\Sigma_{\Upsilon}(x)$ is a $m \times n$ rectangular matrix. The diagonal entries $\sigma_{i}(x)$ of $\Sigma_{\Upsilon}(x)$ are known as the singular values of $\Upsilon(x) . \sigma_{i}(x)$ is listed in descending order at the main diagonal of matrix $\Sigma_{\Upsilon}(x)$. The number of non-zero singular value is equal to the rank $r_{\Upsilon}(x) . \Sigma_{\Upsilon}(x)$ is zero with the exception of the $r_{\Upsilon}(x)$ in the diagonal elements. The non-zero elements in the matrix $\Sigma_{\Upsilon}(x)$ link the left and right singular vectors. The subspace of the linear approximation of the measurement space is spanned by the first $r_{\curlyvee}(x)$ left singular vectors, which is the range of linear approximation of the measurement model mapping to the measurement data. The first $r_{\curlyvee}(x)$ right singular vectors span the subspace of state space, which is linked to the measurement data by the measurement model. When $r_{\curlyvee}(x)<n$, i.e., under-determined cases, the right singular vectors from $r_{\curlyvee}(x)+1$ to $n$ span the subspace of state components, which are not determined and are unobservable from the measurement data.

The pseudo-inverse $\Upsilon^{\dagger}(x)$ is introduced to determine the state observable components with the calculation of $\Sigma \Upsilon^{-1}(x)$ as:

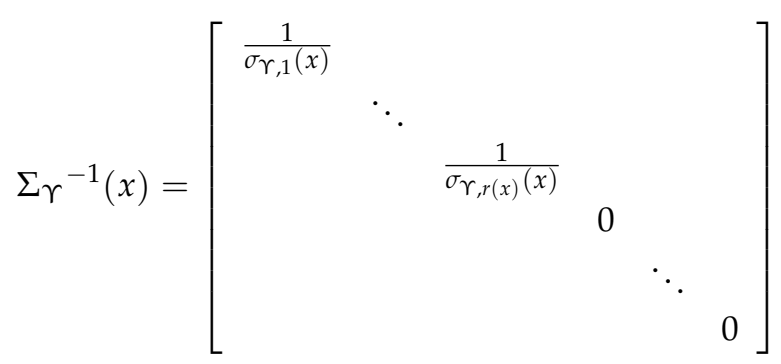

The pseudo-inverse $\Upsilon^{\dagger}(x)$ is defined as Equation (18).

$$
\Upsilon^{\dagger}(x)=U_{\curlyvee}(x) \Sigma_{\Upsilon}^{-1}(x) V_{\Upsilon}^{T}(x)
$$

where $\Upsilon^{\dagger}(x)=\Theta$ is a linear approximation of the invertible space in the adaptive tolerant measurement model. The pseudo-inverse links the measurement data to the observable part of state variables. The iteration step is updated as Equation (19).

$$
\Delta x^{k+1}=\Theta\left(R_{z}+R_{d}\right)^{-1}\left[z-h_{\Sigma, \Delta}(x)^{k}\right]
$$

\section{Measurement Residuals and Observability Analysis}

Using the WLS estimation method, poor data are detected and identified during processing of the measurement residuals. This analysis is essentially based on the properties of these residuals, including their expected probability distribution. The measurement 
residuals using the WLS state estimation method are shown simply below. Consider the measurement equation:

$$
\Delta z=H_{\Sigma, \Delta} \Delta x+e_{\Sigma, \Delta}
$$

where $E\left(e_{\Sigma, \Delta}\right)=0$ and $\operatorname{cov}\left(e_{\Sigma, \Delta}\right)=R$ denoted a diagonal matrix if measurement errors are not correlated. The adaptive WLS estimator of the state vector is obtained as Equation (21).

$$
\begin{aligned}
\Delta \hat{x} & =\left(H_{\Sigma, \Delta}{ }^{T} R^{-1} H_{\Sigma, \Delta}\right)^{-1} H_{\Sigma, \Delta}{ }^{T} R^{-1} \Delta z \\
& =G^{-1} H_{\Sigma, \Delta} R^{-1} \Delta z
\end{aligned}
$$

Then the estimated $\Delta \hat{z}$ is given as:

$$
\Delta \hat{z}=H_{\Sigma, \Delta} \Delta \hat{x}=K \Delta z
$$

where $K=H_{\Sigma, \Delta} G^{-1} H_{\Sigma, \Delta} R^{-1}$. The local measurement redundancy around a given meter can be obtained by checking the corresponding row entries in the matrix $K$. The measurement residual is given as.

$$
\begin{aligned}
r & =\Delta z-\Delta \hat{z} \\
& =(I-K) \Delta z \\
& =(I-K)\left(H_{\Sigma, \Delta} \Delta x+e_{\Sigma, \Delta}\right) \\
& =(I-K) e_{\Sigma, \Delta} \\
& =S e_{\Sigma, \Delta}
\end{aligned}
$$

where $S$ denotes the residual sensitivity matrix. WLS estimation is based on the assumption that the measurement errors follow a Gaussian distribution. According to Equation (24), the probability distribution of the measurement residuals is given as:

$$
\begin{aligned}
E(r) & =E\left(S \cdot e_{\Sigma, \Delta}\right)=0 \\
\operatorname{Cov}(r) & =\Omega=E\left[r r^{T}\right] \\
& =S \cdot E\left[e_{\Sigma, \Delta} e_{\Sigma, \Delta}\right] \cdot S^{T} \\
& =S R S^{T} \\
& =S R
\end{aligned}
$$

Then:

$$
r \sim N(0, \Omega)
$$

The strongly versus weakly interacting measurements can be identified by checking the off-diagonal elements of the residual covariance matrix $\Omega$. Using the condition as $\Omega_{i j} \geq \varepsilon$, then measurement $i$ and $j$ are strongly interacting. Else, these measurements are considered to be weakly interacting or non-interacting. The threshold $\varepsilon$ depends on the network and measurement topology and the desired level of selectivity among measurements.

The observability analysis that determines calculation $\theta(x)$ to be sensitive or not to be sensitive for the changes of unobservable sates $\Delta x$ in the under-determined case. The first-order derivation of $\theta(x)$ is used to analyze the sensitivity, as given in Equation (26).

$$
\left[\frac{\partial \theta(x)}{x}\right]=\left[\frac{\partial \theta(x)}{x_{1}} \cdots \frac{\partial \theta(x)}{x_{2 n}}\right]
$$

The approximation of the changes in $\theta(x)$ with state variation $\Delta x$ is obtained using the Taylor series, as given in Equation (27).

$$
\theta(x+\Delta x) \approx \theta(x)+\left[\frac{\partial \theta(x)}{x}\right] \Delta x
$$


where $\theta(x)$, using the available measurement data, may not be sensitive to all of the state changes caused by unobservable states based on unavailable measurement data. The insensitivities require that the Jacobian vector of $\theta(x)$ is equal to zero for all of the unobservable state changes, as given in Equation (28).

$$
\left[\frac{\partial \theta(x)}{x}\right] \Delta x \stackrel{!}{=} 0
$$

where the equal sign denotes the equation with the condition of insensitivities. The right null space of the measurement Jacobian $\Upsilon(x)$ comprises all of the unobservable state changes, and the elements that are from $(r \curlyvee(x)+1)$ to $n$ in the right singular vectors determine the sensitivity of $\theta(x)$ to unobservable state changes. Therefore, $\theta(x)$ is determined by the available measurement data.

$$
0 !=\left[\frac{\partial \theta(x)}{x}\right]\left[U_{\curlyvee,\left(r_{\curlyvee}(x)+1\right)}(x) \cdots U_{\curlyvee, n}(x)\right]
$$

\section{Simulation}

To demonstrate the adaptive tolerant estimator in mathematics, a distribution network comprising seven nodes was employed to verify the performance of the adaptive tolerant estimator under the condition of model uncertainty in the under-determined case. More complicated cases will be discussed in our future research work.

The input data is shown in Table 1. The measurement configuration of a seven-bus power system is shown in Figure 2. In the under-determined case, the measurement numbers are less than the state numbers. The sign ' $x$ ' denotes that the input data is not provided in under-determined cases.

Table 1. Estimated input data.

\begin{tabular}{cccccccc}
\hline \multirow{2}{*}{$\begin{array}{c}\text { Value } \\
\text { (P. U.) }\end{array}$} & $\mathbf{1}$ & $\mathbf{2}$ & $\mathbf{3}$ & $\mathbf{4}$ & $\mathbf{5}$ & $\mathbf{6}$ & $\mathbf{7}$ \\
\cline { 2 - 8 } & 1.0000 & $\times$ & $\times$ & $\times$ & $\times$ & $\times$ & $\times$ \\
\hline$V_{i} \mid$ & $\times$ & -0.2000 & -0.2000 & -0.2000 & -0.2000 & -0.2000 & -0.2000 \\
\hline$P_{i}$ & $\times$ & 0.0000 & 0.0000 & 0.0000 & 0.0000 & 0.0000 & 0.0000 \\
\hline$Q_{i}$ & $\times$ & $\times$ & $\times$ & $\times$ & $\times$ & $\times$ & $\times$ \\
\hline$P_{i j}$ & $\times$ & $\times$ & $\times$ & $\times$ & $\times$ & $\times$ & $\times$ \\
\hline$P_{j i}$ & $\times$ & $\times$ & $\times$ & $\times$ & $\times$ & $\times$ & $\times$ \\
\hline$Q_{i j}$ & $\times$ & $\times$ & $\times$ & $\times$ & $\times$ & $\times$ & $\times$ \\
\hline$Q_{j i}$ & $\times$ & & & & & &
\end{tabular}

The deviations of lower and upper bound from $\Delta d_{i} i=1,2, \ldots m$. are correspondingly set to be $1 \%$ by the approximation of measurement data. The node voltages are shown in Figure 3 at the bound value of $\varepsilon_{K} \in[-1,1]$.

It can be clearly seen that the real and imaginary parts change with different tendencies at different bound values of $\varepsilon_{K}$. The different values of $\varepsilon_{K}$ represent the different ranges of parameter changes in the model uncertainties.

Figure 4 shows comparative results of the bus voltage phasors using the conventional adaptive and adaptive tolerant estimators. Rapid changes in the parameters lead to model uncertainties. It can be clearly seen that the estimation values produce large deviations and overflow the boundary values. The model uncertainties exaggerate the estimation errors with estimation processing. 


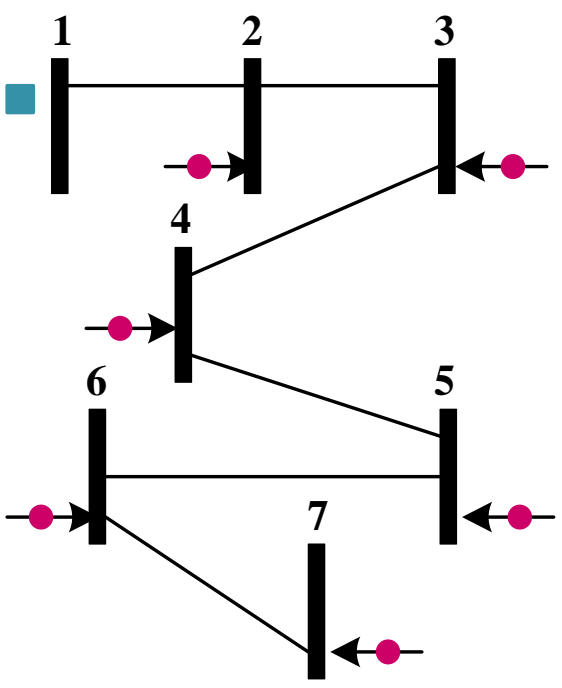

- Voltage magnitude measurement

- Power measurement

Figure 2. Measurement configuration of a seven-bus power systems.

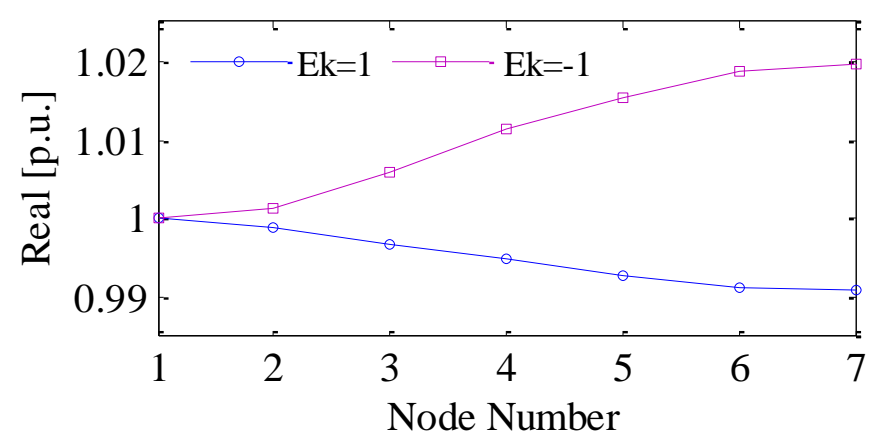

(a)

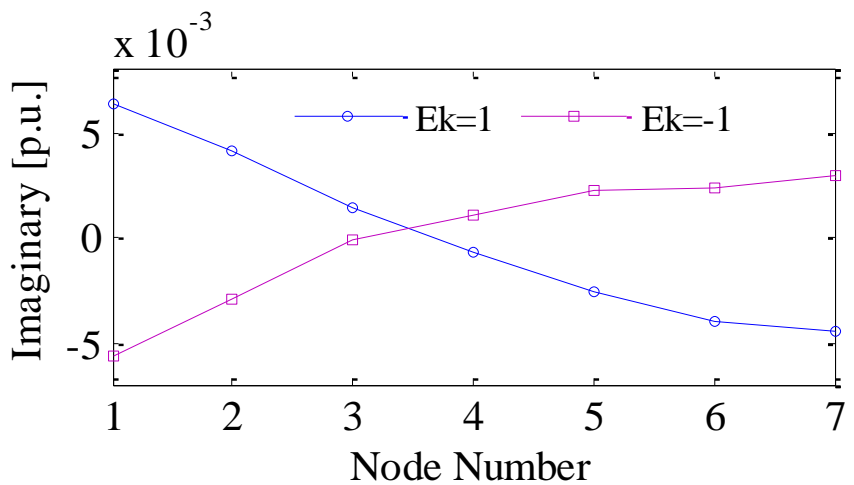

(b)

Figure 3. Bus voltage phasors with (a) real part and (b) imaginary part at the bound values of $\varepsilon_{K}$.

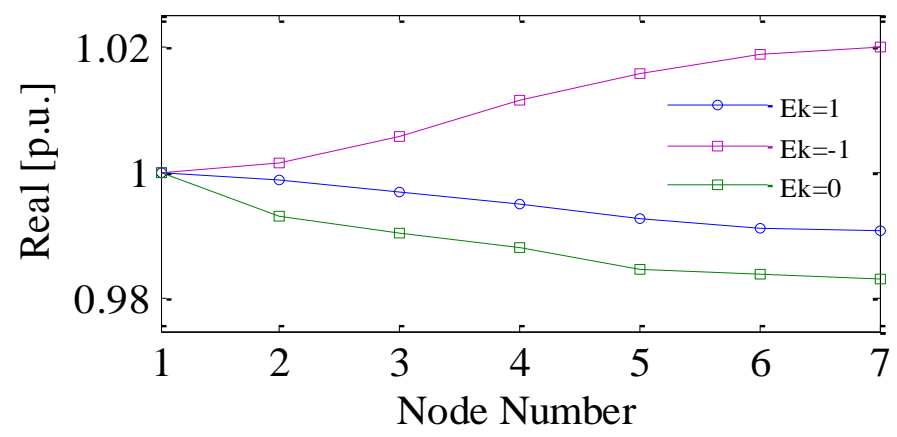

(a)

Figure 4. Cont. 


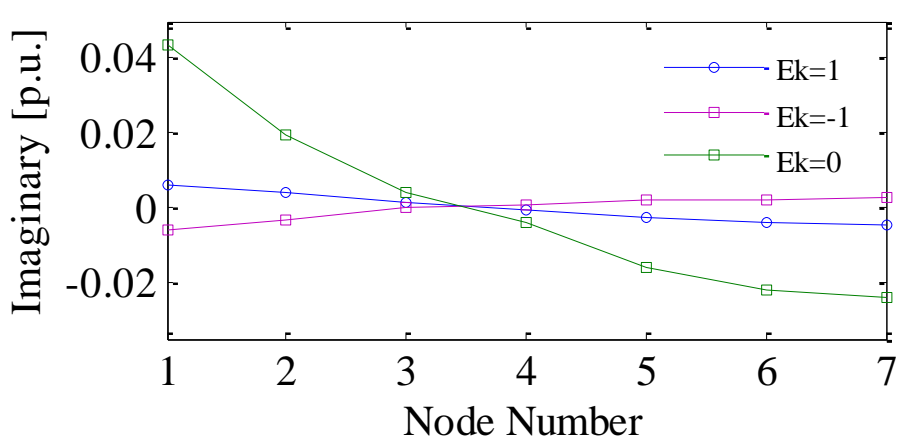

(b)

Figure 4. Bus voltage phasors with (a) real part and (b) imaginary part using conventional adaptive estimator without tolerant estimation.

The estimation results were implemented at $\varepsilon_{K}=-1$ using the adaptive tolerant estimator. Figure 5 shows the comparative results of $\left|V_{i}\right|, P_{i}, Q_{i}$ for estimation and true values using the adaptive tolerant estimator. Although the temperature variations and transmission delay cause rapid changes in the parameters and increase the error of the measurement device, the adaptive tolerant estimator proposed in this paper nonetheless provides accurate estimation of the states at all of the buses and nodes in the power system.

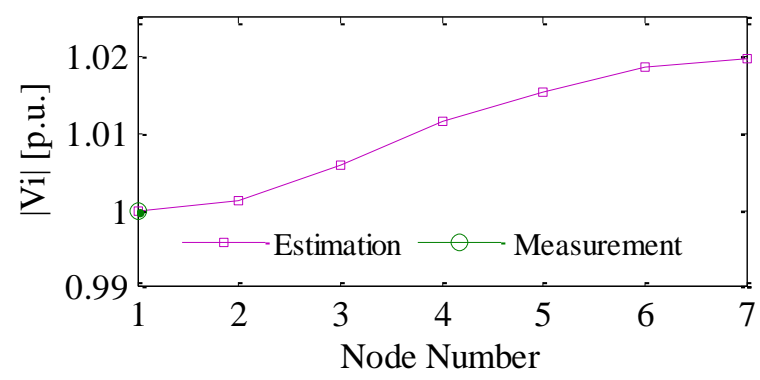

(a)

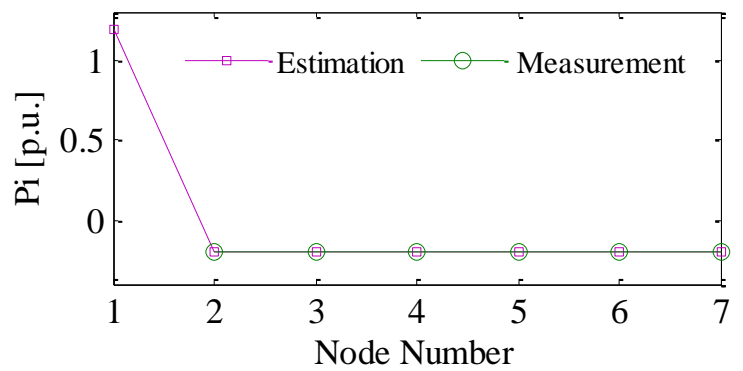

(b)

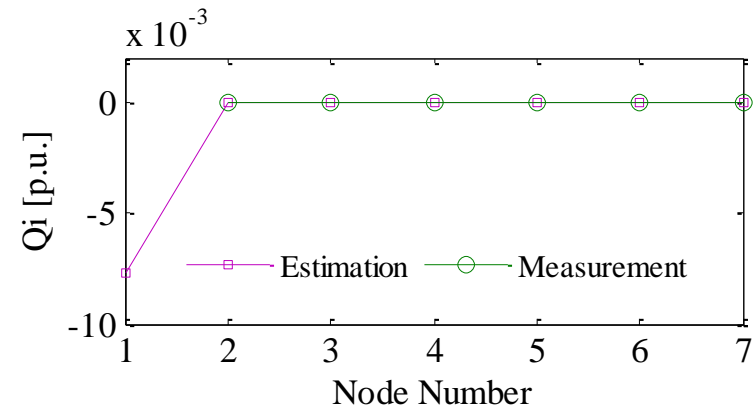

(c)

Figure 5. Estimation and measurement values using the adaptive data tolerant estimator. (a) $\left|V_{i}\right|$; (b) $P_{i} ;$ (c) $Q_{i}$. 
Compared to the original weights in the conventional estimator, the adjusted weights in the adaptive tolerant estimator are displayed in Figure 6. The adjusted weights are larger than the original weights because they comprise the deviations caused by the measurement device errors in the complicated environment. The normal distribution probability of the standard deviation from measurement data is shown in Figure 7. It is noted that a reference line appears in the plot, which is used to judge whether the deviation follows a normal distribution. Although the weights have been adjusted, the deviation roughly conforms to the normal distribution for most nodes in the distribution network.

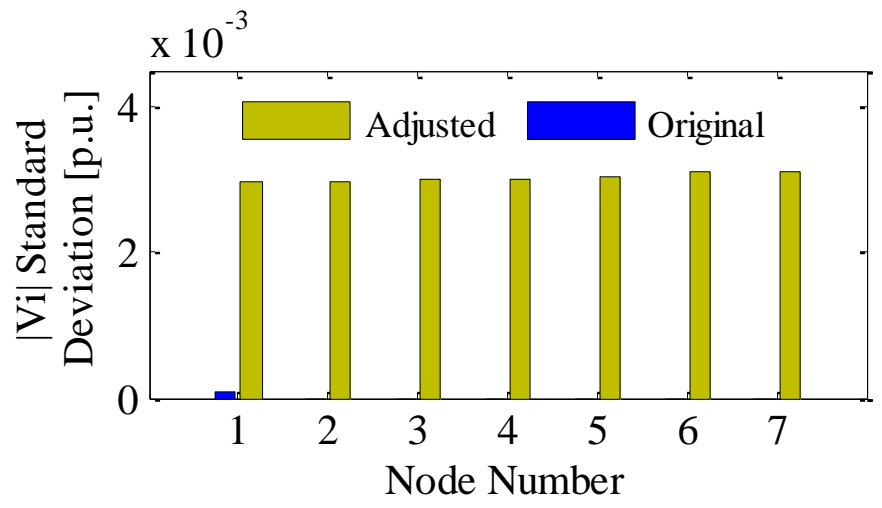

(a)

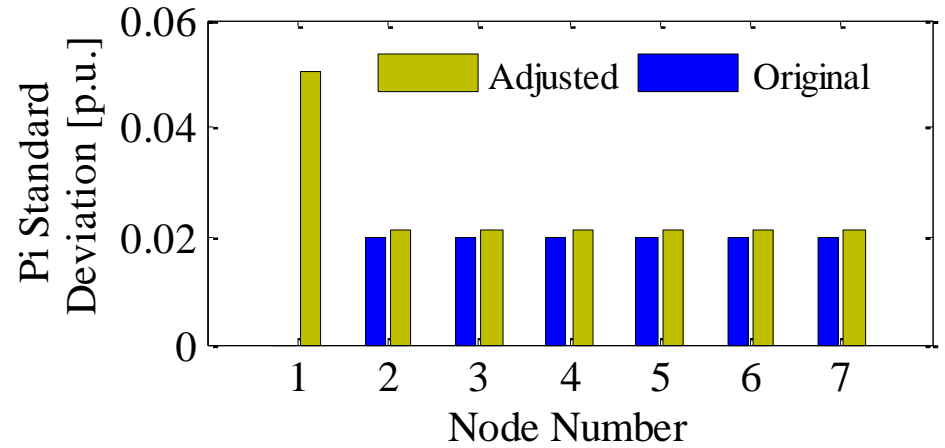

(b)

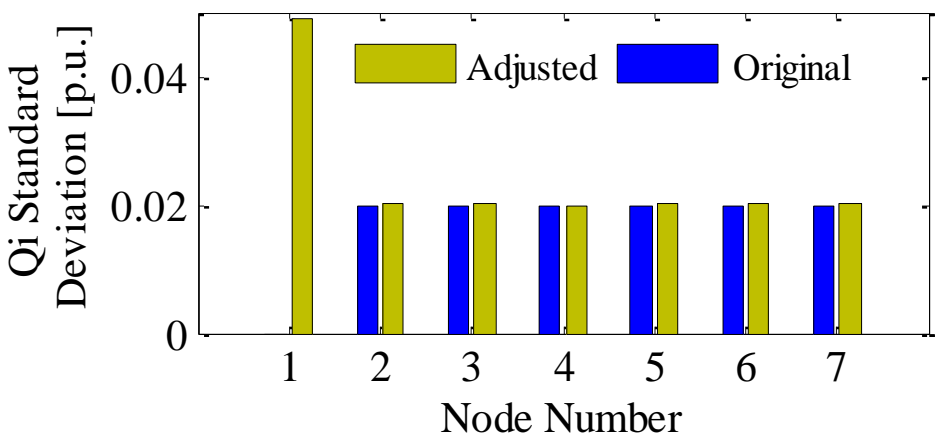

(c)

Figure 6. Original weight in the conventional estimator and adjusted weights in the adaptive data tolerant estimator. (a) $\left|V_{i}\right|$; (b) $P_{i} ;$ (c) $Q_{i}$.

The line estimation results are illustrated in Figure 7, which demonstrates that the adaptive tolerant estimation algorithm can solve the estimation problem in underdetermined cases. The tolerant estimator compensates for the defects of the conventional state estimator based on the WLS, which is only applied to over-determined cases. 


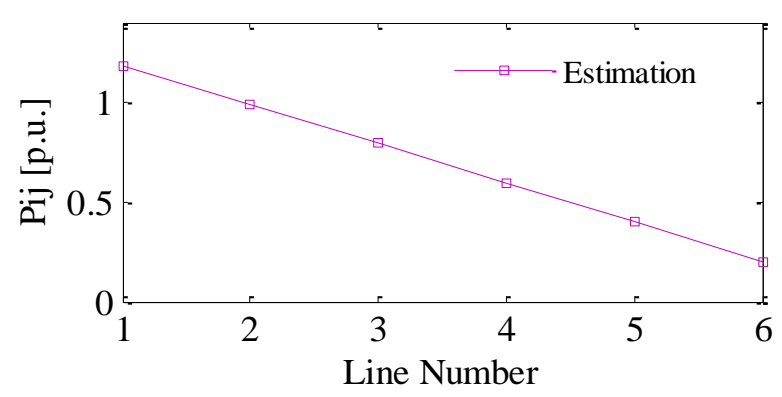

(a)

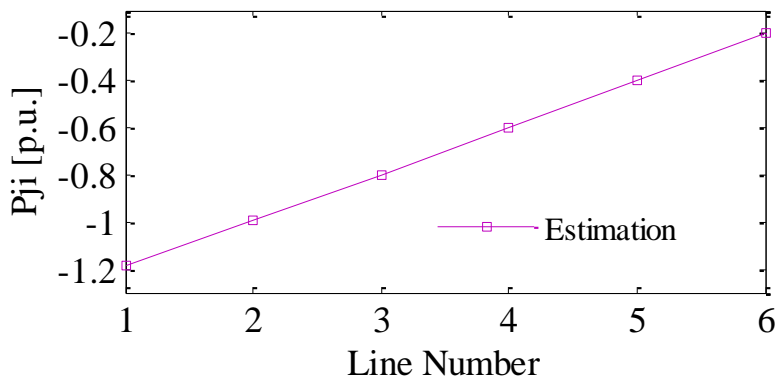

(b)

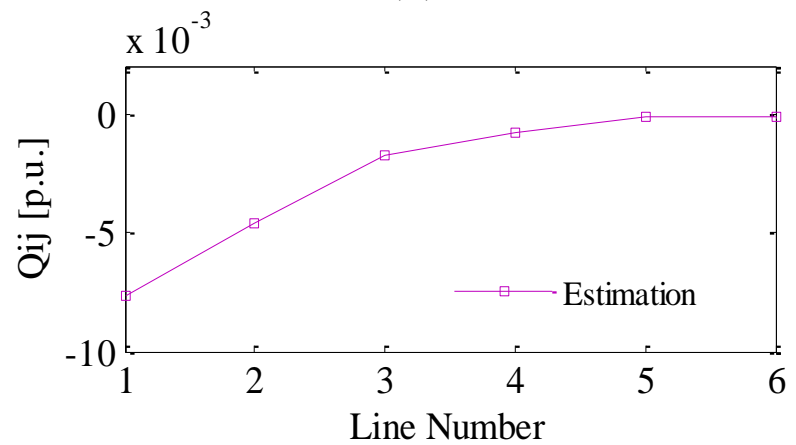

(c)

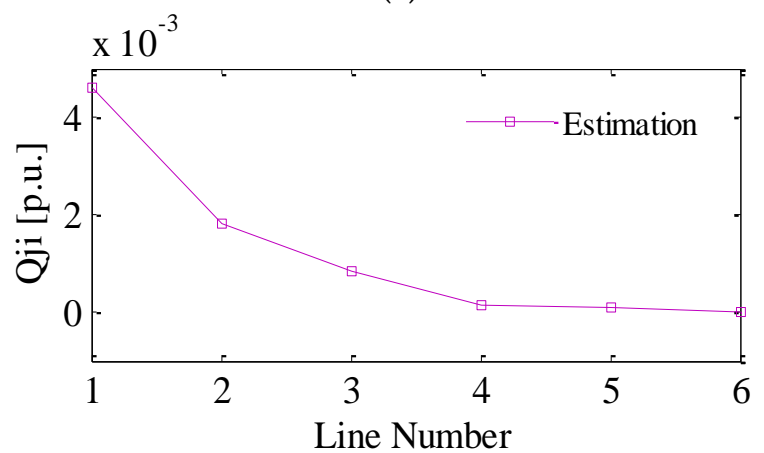

(d)

Figure 7. Line estimation using the adaptive tolerant estimator (a) $P_{i j} ;$ (b) $P_{j i} ;$ (c) $Q_{i j} ;$ (d) $Q_{j i}$.

The comparative results of line estimation using the conventional adaptive estimator and adaptive tolerant estimator are shown in Figure 8 for rapid changes in the parameters.

Compared to the adaptive tolerant estimator, the conventional adaptive estimator increases the line estimation errors under the condition of model uncertainty caused by rapid parameter changes. The errors of line measurement data happen in the $P_{i j}, P_{j i}, Q_{i j}$ and $Q_{j i}$ estimations. The deviations are large for $Q_{i j}$ and $Q_{j i}$, which affects the estimation results and power quality. These deviations remain in the estimation process, causing greater errors in the front of the distribution network, which reduces the estimator performance. 


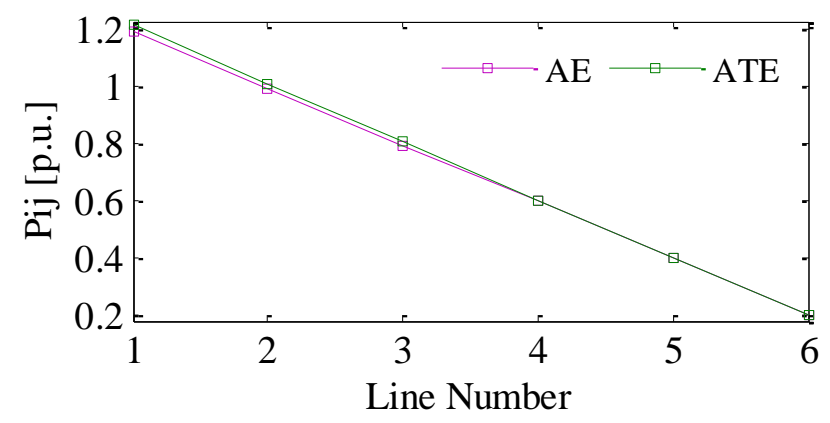

(a)

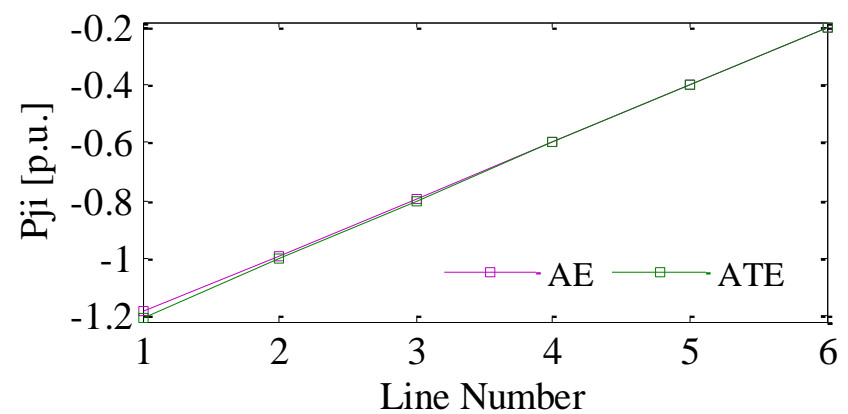

(b)

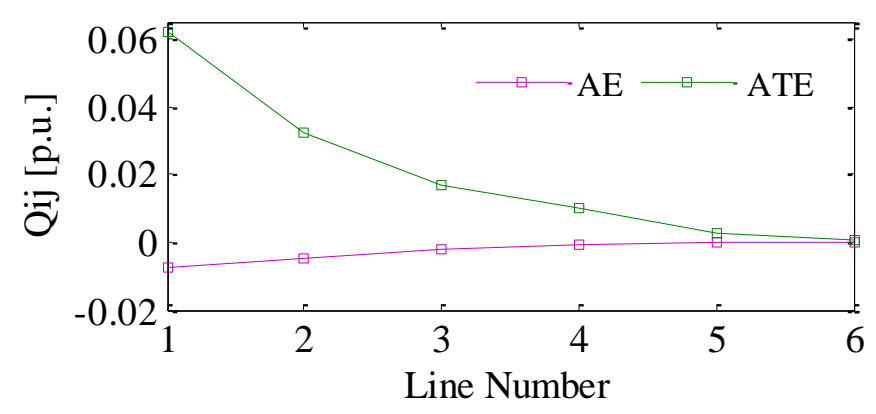

(c)

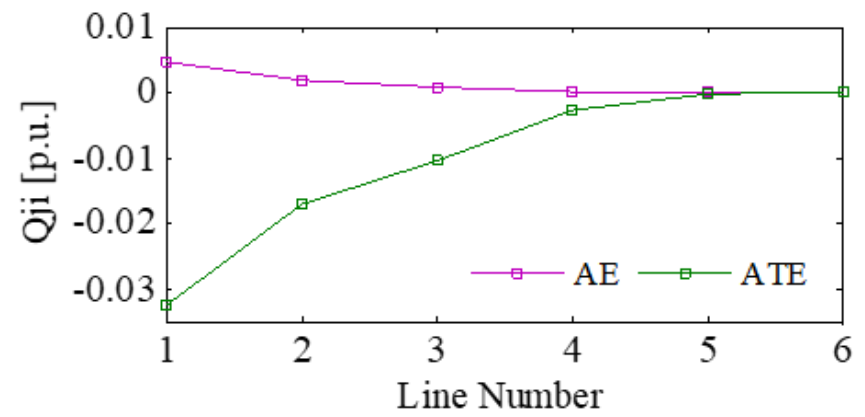

(d)

Figure 8. Line estimation (a) $P_{i j} ;$ (b) $P_{j i} ;$ (c) $Q_{i j}$; (d) $Q_{j i}$ using the conventional adaptive estimator and adaptive tolerant estimator.

\section{Conclusions}

In this paper, a tolerant state estimation algorithm using the SVD method is proposed for distribution network under the condition of model uncertainty. To address the limitations caused by model uncertainty, the design of the tolerant adaptive estimator compensates for the parameter deviations caused by rapid temperature changes and significant transmission delays. This avoids estimation errors caused by model uncertainty. The 
weights of the adaptive tolerant estimator are adjusted to reduce the measurement devices' errors in complicated environments. The adaptive tolerant estimator using the SVD technique extends the over-determined cases to under-determined cases in states that are not fully observable. The adaptive tolerant estimator using the SVD technique realizes accurate state estimations in distribution networks under the condition of measurement model uncertainty. The analysis of observability and residual measurements are demonstrated for the implementation of the algorithm. Compared to the conventional adaptive estimator, the adaptive tolerant estimator reduces estimation errors and provides high-precision estimates under the condition of model uncertainty in complicated environments.

Funding: This research was funded by the National Natural Science Foundation of China, grant number 62003320 and the APC was funded by the National Natural Science Foundation of China.

Informed Consent Statement: Not applicable.

Data Availability Statement: The data used to support the findings of this study are included within this article.

Acknowledgments: This work was supported by the National Natural Science Foundation of China, under Grant 62003320.

Conflicts of Interest: The authors declare that they have no conflict of interest.

\section{References}

1. Yang, X.; Zhang, X.P.; Zhou, S.Y. Coordinated algorithms for distributed state estimation with synchronized phasor measurements. Appl. Energy 2012, 96, 253-260. [CrossRef]

2. Lin, C.H.; Wu, W.C.; Guo, Y. Decentralized Robust state estimation of active distribution grids incorporating microgrids based on PMU measurements. IEEE Trans. Smart Grid 2020, 11, 810-820. [CrossRef]

3. Abur, A.; Expósito, A.G. Power System State Estimation: Theory and Implementation, Electronic Version; CRC Press: Boca Raton, FL, USA, 2004.

4. Wang, G.; Zhu, H.; Giannakis, G.B.; Sun, J. Robust power system state estimation from rank-one measurements. IEEE Trans. Control Netw. Syst. 2019, 6, 1391-1403. [CrossRef]

5. Zhao, J.B.; Netto, M.; Mili, L. A robust iterated extended Kalman Filter for power system dynamic state estimation. IEEE Trans. Power Syst. 2017, 32, 3205-3216. [CrossRef]

6. Zheng, W.Y.; Wu, W.C.; Gomez-Exposito, A.; Zhang, B.M.; Guo, Y. Distributed robust bilinear state estimation for power systems with nonlinear measurements. IEEE Trans. Power Syst. 2017, 32, 499-509. [CrossRef]

7. Tungadio, D.H.; Numbi, B.P.; Siti, M.W.; Jimoh, A.A. Particle swarm optimization for power system state estimation. Neurocomputing 2015, 148, 175-180. [CrossRef]

8. Chavali, P.; Nehorai, A. Distributed power system state estimation Using Factor Graphs. IEEE Trans. Signal Process. 2015, 63, 2864-2876. [CrossRef]

9. Xie, N.; Torelli, F.; Bompard, E.; Vaccaro, A. A graph theory based methodology for optimal PMUs placement and multiarea power system state estimation. Electr. Power Syst. Res. 2015, 119, 25-33. [CrossRef]

10. Risso, M.; Rubiales, A.J.; Lotito, P.A. Hybrid method for power system state estimation. IET Gener. Transmiss. Distrib. 2015, 9, 636-643. [CrossRef]

11. Schweppe, F.C.; Wildes, J. Power system static state estimation-Part I, II \& III. IEEE Trans. Power Apparat. Syst. 1970, 89, 120-125.

12. Abbasi, A.R.; Seifi, A.R. A new coordinated approach to state estimation in integrated power systems. Int. J. Electr. Power Energy Syst. 2013, 5, 152-158. [CrossRef]

13. Cho, Y.S.; Jang, G. New technique for enhancing the accuracy of HVDC systems in state estimation. Int. J. Electr. Power Energy Syst. 2014, 54, 658-663. [CrossRef]

14. Choe, C.; Chen, C.T.; Nagao, S.; Suganuma, K. Real-time acoustic emission monitoring of wear-out failure in SiC power electronic devices during power cycling tests. IEEE Trans. Power Electr. 2021, 36, 4420-4428. [CrossRef]

15. Liu, D.; Zhang, X.; Tse, C.K. A tutorial on modeling and analysis of cascading failure in future power grids. IEEE Trans. Circ. Syst. Ii-Express Briefs 2021, 68, 49-55.

16. Schafer, B.; Witthaut, D.; Timme, M.; Latora, V. Dynamically induced cascading failures in power grids. Nat. Commun. 2018, 9, 13. [CrossRef]

17. Guo, H.D.; Zheng, C.Y.; Iu, H.H.C.; Fernando, T. A critical review of cascading failure analysis and modeling of power system. Renew. Sustain. Energy Rev. 2017, 80, 9-22. [CrossRef]

18. Aien, M.; Rashidinejad, M.; Kouhi, S.; Fotuhi-Firuzabad, M.; Ravadanegh, S.N. Real time probabilistic power system state estimation. Int. J. Electr. Power Energy Syst. 2014, 62, 383-390. [CrossRef] 
19. Arrillaga, J.; Acha, E.; Densem, T.J.; Bodger, P.S. Ineffectiveness of transmission line transpositions at harmonic frequencies. IEE Proc. Part C Gener. Trans. Distrib. 1986, 133, 99-104. [CrossRef]

20. Chen, S.L.; Black, W.Z.; Fancher, M.L. High-temperature sag model for overhead conductors. IEEE Trans. Power Deliv. 2003, 18, 183-188. [CrossRef]

21. Lu, M.L.; Kieloch, Z. Accuracy of transmission line modeling based on aerial LiDAR survey. IEEE Trans. Power Deliv. 2008, 23, 1655-1663. [CrossRef]

22. Madtharad, C.; Premrudeepreechacharn, S.; Watson, N.R. Power system state estimation using singular value decomposition. Electr. Power Syst. Res. 2003, 67, 99-107. [CrossRef]

23. Krause, O.; Martin, D.; Lehnhoff, S. Under-Determined WLMS State Estimation. In Proceedings of the IEEE PES Asia-Pacific Power and Energy Engineering Conference, Brisbane Convention Exhibition Ctr, Brisbane, Australia, 15-18 November 2015.

24. Chen, Y.B.; Ma, J.; Zhang, P.; Liu, F.; Mei, S.W. Robust state estimator based on maximum exponential absolute value. IEEE Trans. Smart Grid 2017, 8, 1537-1544. [CrossRef]

25. Chen, Y.B.; Liu, F.; Mei, S.W.; Ma, J. A robust WLAV state estimation using optimal transformations. IEEE Trans. Power Syst. 2015, 30, 2190-2191. [CrossRef]

26. Zhong, S.; Abur, A. Auto tuning of measurement weights in WLS state estimation. IEEE Trans. Power Syst. 2004, 19, 2006-2013. [CrossRef]

27. Zanella, A.; Chiani, M. Reduced complexity power allocation strategies for MIMO systems with singular value decomposition. IEEE Trans. Veh. Technol. 2012, 61, 4031-4041. [CrossRef]

28. Cai, L.; Thornhill, N.F.; Pal, B.C. Multivariate detection of power system disturbances based on fourth order moment and singular value decomposition. IEEE Trans. Power Syst. 2017, 32, 4289-4297. [CrossRef] 